This item was submitted to Loughborough's Research Repository by the author.

Items in Figshare are protected by copyright, with all rights reserved, unless otherwise indicated.

\title{
Development of new muscle contraction sensor to replace sEMG for using in muscles analysis fields
}

PLEASE CITE THE PUBLISHED VERSION

http://dx.doi.org/10.1109/EMBC.2014.6945225

PUBLISHER

(C) IEEE

VERSION

AM (Accepted Manuscript)

LICENCE

CC BY-NC-ND 4.0

\section{REPOSITORY RECORD}

Zhang, Di, Y. Matsuoka, Weisheng Kong, U. Imtiaz, Luca Bartolomeo, Sarah Cosentino, Massimiliano Zecca, Salvatore Sessa, Hiroyuki Ishii, and Atsuo Takanishi. 2019. "Development of New Muscle Contraction Sensor to Replace Semg for Using in Muscles Analysis Fields". figshare. https://hdl.handle.net/2134/17592. 


\title{
Development of new muscle contraction sensor to replace sEMG for using in muscles analysis fields
}

\author{
D. Zhang, Y. Matsuoka, W. Kong, U. Imtiaz, L. Bartolomeo, Member, IEEE, S. Cosentino, \\ M. Zecca, Member, IEEE, S. Sessa, Member, IEEE, H. Ishii, Member, IEEE, \\ and A. Takanishi, Member, IEEE
}

\begin{abstract}
Nowadays, the technologies for detecting, processing and interpreting bioelectrical signals have improved tremendously. In particular, surface electromyography (sEMG) has gained momentum in a wide range of applications in various fields. However, sEMG sensing has several shortcomings, the most important being: measurements are heavily sensible to individual differences, sensors are difficult to position and very expensive. In this paper, the authors will present an innovative muscle contraction sensing device (MC sensor), aiming to replace SEMG sensing in the field of muscle movement analysis. Compared with sEMG, this sensor is easier to position, setup and use, less dependent from individual differences, and less expensive. Preliminary experiments, described in this paper, confirm that MC sensing is suitable for muscle contraction analysis, and compare the results of SEMG and MC sensor for the measurement of forearm muscle contraction.
\end{abstract}

\section{INTRODUCTION}

In the last two decades, the technologies for detecting, processing and interpreting bioelectrical signals have dramatically improved. In particular surface electromyography (sEMG), a technique for evaluating and recording the electrical activity produced by skeletal muscles, has been more and more employed in a variety of fields, for many different applications. For example, in Neurophysiological and medical research, sEMG, coupled with Inertial Measurement Unit (IMU), has been used to objectively analyze and quantify the movement differences between novice and experts doctors, in tasks like laparoscopic surgery, as a method for objective skill evaluation [1] [2] [3]. In the sport and physiotherapy fields, sEMG is used to

This research has been supported by the JSPS Scientific Research-C grant [24500616], the JSPS Grant-in-Aid for Young Scientists (Wakate B) [25750259], the Global COE Program "Global Robot Academia", MEXT, Japan, and the Consolidated Research Institute for Advanced Science and Medical Care, Waseda University (ASMeW). It was also partially supported by a grant by STMicroelectronics, which also provided the core sensors and the microcontroller.

D. Zhang, Y. Matsuoka, U. Imtiaz, W. Kong and S. Cosentino are with the Graduate School of Advanced Science and Engineering, Waseda University, 3-4-1 Okubo, Shinjuku-ku, Tokyo, 169-8555, Japan

(E-mail: contact@takanishi.mech.waseda.ac.jp, Phone: +81-03-5369-7369 ext: 3604, Fax: +81-03-5269-906).

L. Bartolomeo ,S. Sessa and H. Ishii are with the Global Robot Academia, Waseda University, Tokyo, Japan

M. Zecca is with the School of Electronic, Electrical and Systems Engineering, Loughborough University, Leics LE11 3TU, UK.

A. Takanishi is with the Department of Modern Mechanical Engineering, Waseda University, and Humanoid Robotics Institute, Waseda University, Tokyo, Japan. monitor the effectiveness of strength or endurance training or sport injury rehabilitation [4] [5]. sEMG is even used in Ergonomics to analyze posture and help in the design of workplaces and in the early detection of posture disorder development [6] [7]. The physiological analysis of movement via sEMG has been extended also to the animal world, even to the insects [8] [9]. Hence, sEMG has become a proven measurement technique in a large variety of fields and applications.

The authors themselves also relied on sEMG sensing to analyze human motion in various different applications. However, the sEMG sensing technique presents several shortcomings. First of all, sEMG sensors must be fixed in position with tight bands or seals to avoid any undesired shifting of the skin-electrode point of contact. The subject movement causes the skin-electrode interface to change, leading to variation in the electrodes contact resistance and in the polarization potential. This problem leads to a substantial increase of the time for sEMG sensor positioning and setup. In addition, even if the sEMG is tightly fixed, the measured value differs significantly depending on the effective position of the sensor. Furthermore, in order to obtain reliable measurements and reduce inter-subject individual differences in values, subjects should be shaved in all the sensing area. Moreover, sEMG sensor measurement can be affected by temperature and humidity, and by muscles condition. At last, commercially available sEMG systems are expensive.

The authors decided then to implement an innovative sensing technique able to overcome all the mentioned problems. In this paper a new muscle contraction sensor is presented, named Mechanical Contraction (MC) sensor, to substitute sEMG sensors. Compared to sEMG sensors, the $\mathrm{MC}$ sensor is easier to setup, more reliable and cheaper than commercial sEMG sensors. To verify these claims, the authors developed a MC sensing system for the forearm, and run experiments to compare the performances of both sEMG and $\mathrm{MC}$ forearm muscle contraction measurement techniques.

The structure of this paper is as follows: Section II, Materials and methods, is divided in two subsections. In the first subsection, the authors will describe in detail the sensor basic principle and hardware implementation; in the second, the protocol used for the evaluation experiments. Section III, Results and Discussion, will present and discuss the results obtained in the experiments. The last section, conclusions, will recapitulate the major lines of the paper and will present ideas for future applications of the newly developed sensing technique. 


\section{MATERIALS AND METHODS}

\section{A. Sensor Principle}

In general, we can extract three different types of data during muscle movement. The first type is the muscle surface electric potential change, another one is the muscle surface curvature change, and the last type is the muscle contraction change. The first type of data is measured with sEMG, the second type is not reliable because it is heavily affected by individual differences. Therefore, we focused on measuring the muscle contraction change. In Fig.1, the sensing principle of the new muscle contraction sensor is shown.

In Fig.1, four different elastic moduli are can be seen. $K_{\text {band }}$, $K_{\text {spring }}, K_{\text {stick}}$, are respectively the elastic modulus of the sensor band, spring, and stick, whilst $K_{\text {muscle }}$ is the elastic modulus of the muscle.

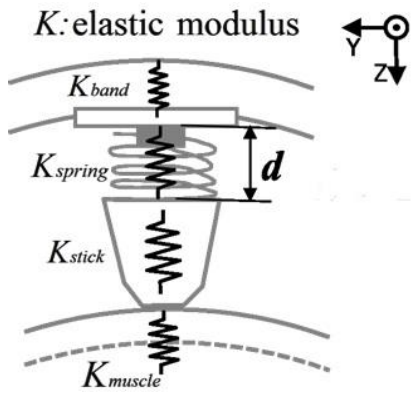

Figure 1. Measurement principle

In particular, $K_{\text {muscle }}$ changes depending on the muscle state. Therefore, we can distinguish another two elastic moduli: $K_{\text {relax }}$ and $K_{\text {contraction, }}$, respectively the elastic module of the muscle relaxation state and the muscle contraction state.

As the band and the sensor stick are made of solid material, we can consider $K_{\text {band }}$ and $K_{\text {stick }}$ much bigger than the other elastic moduli. If we choose then a spring with a $K_{\text {spring }}$ between $K_{\text {relax }}$ and $K_{\text {contraction }}$, we can observe two different Situations:

- muscle in relaxation state: the spring stiffness is higher than the muscle stiffness, the spring is naturally extended

- muscle in contraction state: the spring stiffness is lower than the muscle stiffness, the spring is naturally compressed

Hence, muscle contraction and relaxation cause $K_{\text {muscle }}$ to change from $K_{\text {relax }}$ to $K_{\text {contraction, }}$ resulting in a linear deformation of the spring, $\Delta d$. Measuring $\Delta d$ we can infer the muscle stiffness $\Delta K_{\text {muscle }}$ and estimate the muscle contraction.

The relationship between the elastic moduli is defined in (1):

$$
K_{\text {band }}, K_{\text {stick }}>>K_{\text {contraction }}>K_{\text {spring }}>K_{\text {relax }}
$$

\section{B. Sensor Hardware}

To verify the validity of this sensing technique, the authors designed and implemented an MC sensor band for forearm muscle contraction measurement. A sensor row unit is shown in Fig.2. One sensor row is composed of four springs, to obtain more precise information on the muscles movement, fixed to a Printed Circuit Board (PCB). The space between the springs is $15 \mathrm{~mm}$; the height of one element is $10 \mathrm{~mm}$ in full extension.

To measure the spring deformation, a photo reflector (SG105) is set in the center of each sensor element, on the PCB.

Several springs with different stiffness constants have been then compared to determine the most suitable $K_{\text {spring }}$; and a peak performance has been achieved with a stiffness constant of $\mathrm{K}=2.0[\mathrm{~N} / \mathrm{mm}]$

The MC sensor has been designed to simultaneously measure the contraction of all the muscles of the forearm. Given the standard perimeter of a human's forearm, eight rows and 32 springs have been implemented. The distance between two rows is $30 \mathrm{~mm}$ and the height of the MC sensor row is $210 \mathrm{~mm}$. The sensors transmit data to a central board via CAN bus, and the central board transmits the grouped data via Bluetooth to a remote PC for data storage and analysis. The full sensor band is shown in Fig.3.

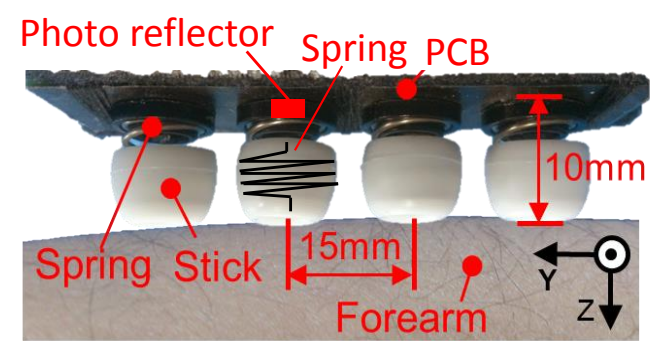

Figure 2. One row of the new muscle contraction sensor

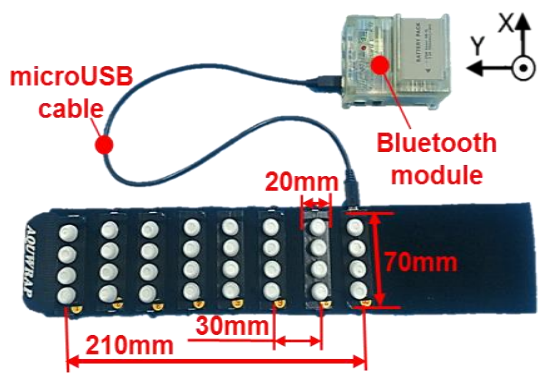

Figure 3. Overview of the new muscle contraction sensor

\section{Protocol}

The sensor performance evaluation experiment was carried out with eight subjects, male, right handed. Each subject performed the below described experiments three times. All the experiments have been video recorded.

The right to privacy of the subjects has been fully respected. In accordance with the ethical standard defined by the committee of Waseda University and with the ethical standards laid down in the 1964 Declaration of Helsinki and its later amendments, the subjects have been extensively informed about their rights and asked to sign a written form for the consent to the experiment. 


\section{Experiment I: Correlation between muscle stiffness and muscle contraction}

As mentioned above, measuring the linear deformation $\Delta d$ of the spring during muscle contraction the muscle stiffness $\Delta K_{\text {muscle }}$ can be inferred, and from it the muscle contraction can be estimated. However, in order to do so, the correlation between muscle stiffness and muscle contraction must be clearly identified. Discover the numerical correlation between muscle stiffness and contraction was the aim of this first experiment.

In this experiment, control data from a dynamometer and a muscle durometer (NEUTONE TDM-N1/NA1) are recorded.

Subjects held the dynamometer in the right hand. To validate the correlation between muscle hardness and muscle contraction, the subject's arm must be fixed in position and the joints must maintain fixed angles. At first, the subject was asked to contract the forearm at the maximum of their capability, and using the dynamometer, their maximum developed force was recorded. Then, the subject was asked to contract the forearm at $10 \%$ of their previously recorded maximum force, while the current muscles hardness is measured with a muscle durometer. Eventually, the subject was asked to contract the forearm at from zero to the maximum force by steps of $10 \%$ of their previously recorded maximum force, and data from both the dynamometer and the muscle durometer were recorded. The measurement unit of the muscle durometer is Tone.

\section{E. Experiment II: Performance between sEMG and the new muscle contraction sensor}

In this experiment, control data from the dynamometer and WB-EMG are recorded [10]. Comparing the performances between MC and sEMG sensor was the aim of this second experiment.

In Fig.4, the schematic diagram of forearm muscles is shown. The muscle from which the level of muscle contraction is computed is colored in red. The muscle responsible for the hand movement is colored in yellow. The muscles from which the muscle force is computed are colored in blue [11]. In this experiment, the focus was on the Flexor Carpi Radialis (L-FCR).

The subject was asked to grip the dynamometer using $100 \%$ of their strength, for three consecutive times. Then the subject was allowed to rest for 5 minutes to recover from muscle fatigue. After that, the subject was asked to grip the dynamometer 3 times using 75\% strength, 50\% strength and

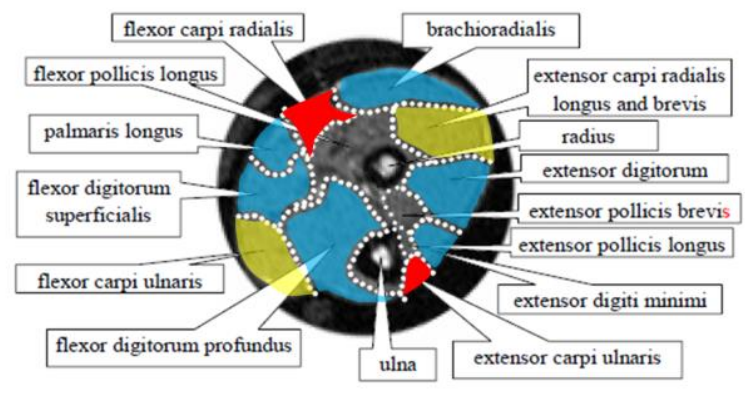

Figure 4. Schematic diagram of the forearm muscle
$25 \%$ strength.

Comparing the performances of sEMG and MC sensor is difficult because the ranges of data values of the measured parameters are significantly different. Therefore, both data from sEMG and MC sensors have been normalized before the comparison, according to this simple transformation:

$$
S_{m}(k)=\frac{1}{\delta^{2}}\left(L_{m}(k)-\mu_{m}\right)
$$

where

$$
\begin{gathered}
\mu_{m}=\frac{1}{N} \sum_{k=1}^{N} L_{m}(k) \\
\delta_{m}{ }^{2}=\frac{1}{N} \sum_{k=1}^{N}\left(L_{m}(k)-\mu_{m}\right)
\end{gathered}
$$

And where $L_{m}(k)$ is the sample. $N$ is the total number of subjects.

\section{RESULTS AND DISCUSSION}

Results of Experiment I are shown in Fig.5.

In Fig.5, the correlation function between muscle hardness and muscle activation of eight subjects are shown. The function is linear, as expected, so we can safely estimate the muscle activation by measuring the muscle hardness.

In Table I, the computed correlation coefficients of all the eight subjects are shown. Based on the results on these eight subjects, the average correlation coefficient between muscle hardness and muscle contraction is 0.983 .

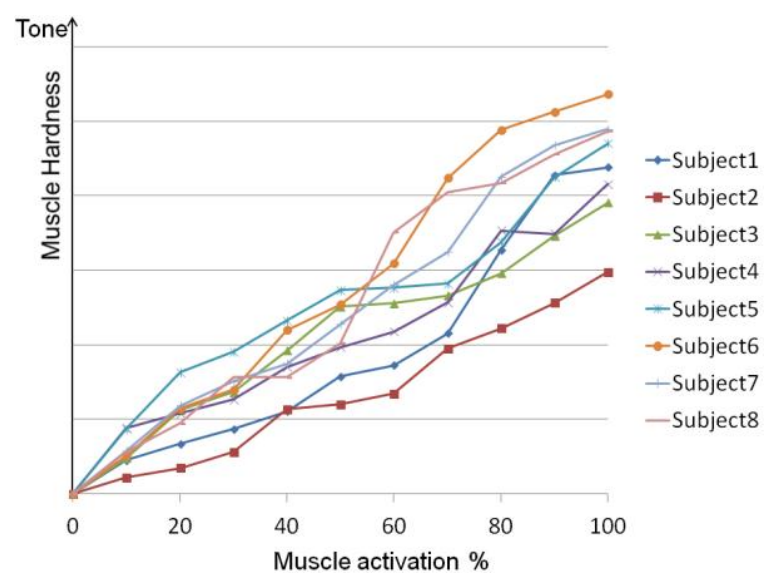

Figure 5. Correlation of forearm muscle contraction and hardness

TABLE I. CORRELATION COEFFICIENT OF THE MUSCLE HARDNESS AND MUSCLE CONTRACTION

\begin{tabular}{|c|c|c|c|c|}
\hline Subject & $\boldsymbol{0 1}$ & $\boldsymbol{0 2}$ & $\boldsymbol{0 3}$ & $\boldsymbol{0 4}$ \\
\hline R & 0.973 & 0.989 & 0.986 & 0.985 \\
\hline Subject & $\boldsymbol{0 5}$ & $\boldsymbol{0 6}$ & $\boldsymbol{0 7}$ & $\boldsymbol{0 8}$ \\
\hline $\mathbf{R}$ & 0.975 & 0.993 & 0.994 & 0.983 \\
\hline
\end{tabular}

Results of Experiment II are shown in Fig.6 and Fig.7.

In Fig. 6, the performance of both the sEMG and the MC sensor when the muscle is exerting $100 \%$ of its strength are shown. The performances of the two different sensing systems appear very similar, as expected. 
In Fig.7, the performance of both the sEMG and the MC sensor when the muscle is contracted at $75 \%, 50 \%$, and $25 \%$ of the maximum individual strength is shown. Again, even if on MC sensor signal a delay can be observed, because the myoelectricity signal is faster than the muscle activity, overall performances are very similar. We computed the correlation coefficient of two lines is 0.894 .

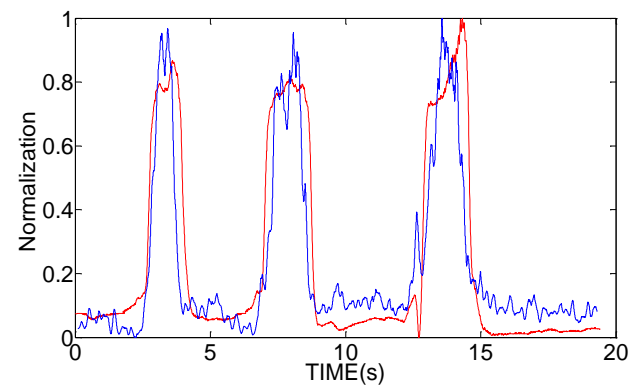

Figure 6. Performance of sEMG and new muscle contraction sensor (100\% strength)

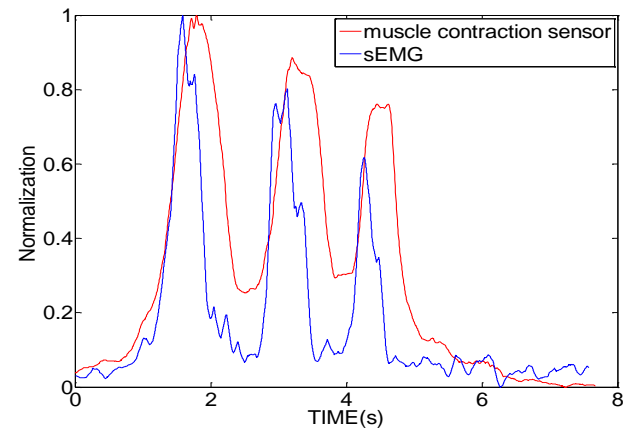

Figure 7. Performance of sEMG and new muscle contraction sensor ( $75 \%, 50 \%$ and $25 \%$ maximum strength)

These results demonstrate that MC based sensing systems can be used for muscles state analysis and muscle contraction measurement, and can replace the much more expensive sEMG based sensing systems. In Table II, the comparison of usability between MC sensor and sEMG is shown.

TABLE II. COMPARISON OF USABILITY BETWEEN MC SENSOR AND SEMG

\begin{tabular}{|c|c|c|}
\hline & sEMG & MC sensor \\
\hline Preparation time[s] & 360 & 48 \\
\hline Time to wear[s] & 63 & 14 \\
\hline Total cost $[\mathbf{\$}]$ & 20,000 & 1,600 \\
\hline
\end{tabular}

\section{CONCLUSION}

In this paper, the authors developed a new muscle contraction sensor to replace the sEMG in muscles movement analysis and muscle contraction measurement. Compared to the sEMG, the new sensor is easier to set up, easier to wear and cheaper. In addition, the authors run experiment to confirm that the new sensor can be used for its designed application, and compared the performances of the new sensor and the sEMG.

Future works in the field will develop in two different directions. One is that use the MC sensor to do the specific experiments to compare the performance between the MC sensor and sEMG. The other is that improve the sensor so that not only judge the muscles state but also compute the state of the muscles.

\section{ACKNOWLEDGMENT}

This research has been conducted at the Waseda University Center for Advanced Biomedical Sciences, TWIns. The authors would like to express their thanks to the Italian Ministry of Foreign Affairs, General Directorate for Cultural Promotion and Cooperation, for its support to RoboCasa. The authors would also like to express their gratitude to Life Performance Research, Okino Industries LTD, Japan ROBOTECH LTD, SolidWorks Corp, Dyden, Kyoto Kagaku Co. Ltd. for their support to the research.

\section{REFERENCES}

[1] L. Bartolomeo, Y. Noh, Y. Kasuya, M. Nagai, M. Zecca, S. Sessa, S. Cosentino, K. Saito, Z. Lin, H. Ishii, and A. Takanishi, "Biomechanical Evaluation of the Phases during Simulated Endotracheal Intubation (ETI): Pilot Study on the Effect of Different Laryngoscopes", EMBC, 2013.

[2] Y. Matsuoka, L. Bartolomeo, T. Chihara, U. Imtiaz, K. Saito, W. Kong, Y. Noh, Y. Kasuya, M. Nagai, M. Ozaki, C. Wang, S. Sessa, H. Ishii, M. Zecca and A. Takanishi, "A Novel Approach to Evaluate Skills in Endotracheal Intubation Using Biomechanical Measurement System", ROBIO, 2013.

[3] S. Sessa, M. Zecca, Z. Lin, L. Bartolomeo, H. Ishii, and A. Takanishi, "A Methodology for the Performance Evaluation of Inertial Measurement Units," Journal of Intelligent \& Robotic Systems, pp. $1-15,2012$.

[4] Yoo-hyun KIM, Seo-jun KIM, Hyeon-min SHIM, Sang-min LEE, Kyu-sung Kim, "A Method for Gait Rehabilitation Training using EMG Fatigue Analysis", ICTC, 2013.

[5] A. Holtermann, P.J. Mork, L.L. Andersena, H.B. Olsena, K. Søgaard, "The use of EMG biofeedback for learning of selective activation of intra-muscular parts within the serratus anterior muscle A novel approach for rehabilitation of scapular muscle imbalance," Journal of Electromyography and Kinesiology, pp. 359-365, 2010

[6] Goran M. Hagg, Alwin Luttmann, Matthias Jager, "Methodologies for evaluating electromyographic field data in ergonomics," Journal of Electromyography and Kinesiology, pp. 301-312, 2000.

[7] Svend Erik Mathiassen, Alex Burdorf, Allard J.van der Beek, "Statistical power and measurement allocation in ergonomic intervention studies assessing upper trapezius EMG amplitude A case study of assembly work," Journal of Electromyography and Kinesiology, pp. 45-57, 2002.

[8] M. Nagaraju, J. Silver, F. Zhang, Y. Liao, J. Pandey, T. Morrison, A. Mishra, D. Yeager, and B. Otis, "Circuit techniques for wireless bioelectrical interfaces," in 2010 International Symposium on VLSI Design Automation and Test (VLSI-DAT), 2010, pp. 117 -120.

[9] R. R. Harrison, H. Fotowat, R. Chan, R. J. Kier, R. Olberg, A. Leonardo, and F. Gabbiani, "Wireless Neural/EMG Telemetry Systems for Small Freely Moving Animals," IEEE Transactions on Biomedical Circuits and Systems, vol. 5, no. 2, pp. 103 -111, Apr. 2011.

[10] U. Imtiaz, L. Bartolomeo,Z. Lin, S. Sessa, H. Ishii, K. Saito, M. Zecca and A. Takanishi, "Design of a Wireless Miniature Low cost EMG Sensor using Gold Plated Dry Electrodes for Biomechanics research", ICMA, 2013.

[11] Yukari Tanikawa, Takamine, Michio Miyakawa, Kiryu, Kizuka Asa-haku, Endo Yasuomi, Shinpei Okawa, Yukio Yamada, "Evaluation of the human forearm muscle activity by diffuse optical tomography", Institute of Electrical Engineers Journal C (Electronics, Information and Systems department magazine)Vol. 132 (2012) No. 3 P 374-383 